\title{
Stability of $n$-Jordan Homomorphisms from a Normed Algebra to a Banach Algebra
}

\author{
Yang-Hi Lee \\ Department of Mathematics Education, Gongju National University of Education, Gongju 314-711, Republic of Korea \\ Correspondence should be addressed to Yang-Hi Lee; yanghi2@hanmail.net
}

Received 14 May 2013; Revised 7 August 2013; Accepted 3 September 2013

Academic Editor: Janusz Brzdęk

Copyright (C) 2013 Yang-Hi Lee. This is an open access article distributed under the Creative Commons Attribution License, which permits unrestricted use, distribution, and reproduction in any medium, provided the original work is properly cited.

We establish the hyperstability of $n$-Jordan homomorphisms from a normed algebra to a Banach algebra, and also we show that an $n$-Jordan homomorphism between two commutative Banach algebras is an $n$-ring homomorphism.

\section{Introduction}

Let $A, B$ be two rings (algebras) and $n$ a positive integer greater than 1 . An additive mapping $g: A \rightarrow B$ is called an $n$-Jordan homomorphism if $g\left(a^{n}\right)=(g(a))^{n}$ for all $a \in A$ and an additive mapping $h: A \rightarrow B$ is called an $n$-ring homomorphism if $h\left(\prod_{i=1}^{n} a_{i}\right)=\prod_{i=1}^{n} h\left(a_{i}\right)$ for all $a_{1}, a_{2}, \ldots$, $a_{n} \in A$.

In 2009, Gordji et al. [1] showed the following theorems.

Theorem 1. Let $n \in\{2,3,4,5\}$ be fixed. Suppose that $A, B$ are two commutative algebras. Let $h: A \rightarrow B$ be an $n$-Jordan homomorphism. Then $h$ is an n-ring homomorphism.

Theorem 2. Let $n \in\{2,3,4,5\}$ be fixed. Suppose that $A, B$ are commutative Banach algebras. Let $\delta$ and $\varepsilon$ be nonnegative real numbers, and let $p, q$ be real numbers such that $(p-1)(q-1)>$ $0, q \geq 0$ or $(p-1)(q-1)>0, q<0$, and $f(0)=0$. Assume that $f: A \rightarrow B$ satisfies the system of functional inequalities:

$$
\begin{gathered}
\|f(a+b)-f(a)-f(b)\| \leq \varepsilon\left(\|a\|^{p}+\|b\|^{p}\right), \\
\left\|f\left(a^{n}\right)-f(a)^{n}\right\| \leq \delta\|a\|^{n q},
\end{gathered}
$$

for all $a, b \in A$. Then, there exists a unique n-ring homomorphism $h: A \rightarrow B$ such that

$$
\|f(a)-h(a)\| \leq \frac{2 \varepsilon}{\left|2-2^{p}\right|}\|a\|^{p},
$$

for all $a \in A$.
The stability problem of group homomorphisms was formulated by Ulam [2] in 1940. Bourgin [3] and Badora [4] solved the stability problem of ring homomorphisms (see [5]). The term hyperstability was used for the first time in [6]. Some recent results on hyperstability of Cauchy or linear equation can be founded in $[5,7,8]$.

In this paper, we improve Theorems 1 and 2 into Theorems 4 and 8 , respectively. In particular, we prove the hyperstability of $n$-Jordan homomorphisms between two commutative Banach algebras.

\section{Generalization of Theorem 1}

Lemma 3. Let $n, k$ be fixed natural numbers with $n>k \geq 2$. Let $A, B$ be two commutative algebras, and let $f: A \rightarrow B$ be an additive mapping. Assume that $f$ satisfies the following equality:

$$
\begin{gathered}
\sum_{i_{1}=k-1}^{n-1} \sum_{i_{2}=k-2}^{i_{1}-1} \ldots \sum_{i_{k-1}=1}^{i_{k-2}-1}\left(\begin{array}{l}
n \\
i_{1}
\end{array}\right)\left(\begin{array}{l}
i_{1} \\
i_{2}
\end{array}\right) \cdots\left(\begin{array}{l}
i_{k-2} \\
i_{k-1}
\end{array}\right) \\
\times f\left(x_{1}^{n-i_{1}} x_{2}^{i_{1}-i_{2}} x_{3}^{i_{2}-i_{3}} \cdots x_{k}^{i_{k-1}}\right) \\
=\sum_{i_{1}=k-1}^{n-1} \sum_{i_{2}=k-2}^{i_{1}-1} \cdots \sum_{i_{k-1}=1}^{i_{k-2}-1}\left(\begin{array}{l}
n \\
i_{1}
\end{array}\right)\left(\begin{array}{l}
i_{1} \\
i_{2}
\end{array}\right) \cdots\left(\begin{array}{l}
i_{k-2} \\
i_{k-1}
\end{array}\right) f\left(x_{1}\right)^{n-i_{1}} \\
\times f\left(x_{2}\right)^{i_{1}-i_{2}} \cdots f\left(x_{k}\right)^{i_{k-1}},
\end{gathered}
$$


for all $x_{1}, x_{2}, x_{3}, \ldots, x_{k} \in A$. Then one gets

$$
\begin{aligned}
& \sum_{i_{1}=k}^{n-1} \sum_{i_{2}=k-1}^{i_{1}-1} \cdots \sum_{i_{k}=1}^{i_{k-1}-1}\left(\begin{array}{c}
n \\
i_{1}
\end{array}\right)\left(\begin{array}{c}
i_{1} \\
i_{2}
\end{array}\right) \cdots\left(\begin{array}{c}
i_{k-1} \\
i_{k}
\end{array}\right) \times f\left(x_{1}^{n-i_{1}} x_{2}^{i_{1}-i_{2}} \cdots x_{k+1}^{i_{k}}\right) \\
&=\sum_{i_{1}=k}^{n-1} \sum_{i_{2}=k-1}^{i_{1}-1} \cdots \sum_{i_{k}=1}^{i_{k-1}-1}\left(\begin{array}{c}
n \\
i_{1}
\end{array}\right)\left(\begin{array}{c}
i_{1} \\
i_{2}
\end{array}\right) \cdots\left(\begin{array}{c}
i_{k-1} \\
i_{k}
\end{array}\right) f\left(x_{1}\right)^{n-i_{1}} \\
& \times f\left(x_{2}\right)^{i_{1}-i_{2}} \cdots f\left(x_{k+1}\right)^{i_{k}},
\end{aligned}
$$

for all $x_{1}, x_{2}, x_{3}, \ldots, x_{k+1} \in A$.

Proof. Replacing $x_{k}$ by $x_{k+1}$ in (3), we obtain

$$
\begin{aligned}
& \sum_{i_{1}=k-1}^{n-1} \ldots \sum_{i_{k-1}=1}^{i_{k-2}-1}\left(\begin{array}{c}
n \\
i_{1}
\end{array}\right) \ldots\left(\begin{array}{l}
i_{k-2} \\
i_{k-1}
\end{array}\right)\left(\begin{array}{c}
i_{k-1} \\
i_{k-1}
\end{array}\right) \\
& \times f\left(x_{1}^{n-i_{1}} \cdots x_{k-1}^{i_{k-2}-i_{k-1}} x_{k+1}^{i_{k-1}}\right) \\
&=\sum_{i_{1}=k-1}^{n-1} \cdots \sum_{i_{k-1}=1}^{i_{k-2}-1}\left(\begin{array}{l}
n \\
i_{1}
\end{array}\right) \cdots\left(\begin{array}{c}
i_{k-2} \\
i_{k-1}
\end{array}\right)\left(\begin{array}{c}
i_{k-1} \\
i_{k-1}
\end{array}\right) \\
& \times f\left(x_{1}\right)^{n-i_{1}} \ldots f\left(x_{k-1}\right)^{i_{k-2}-i_{k-1}} \\
& \times f\left(x_{k+1}\right)^{i_{k-1}},
\end{aligned}
$$

for all $x_{1}, x_{2}, x_{3}, \ldots, x_{k-1}, x_{k+1} \in A$. In particular, the equality (3) implies that

$$
\begin{aligned}
& \sum_{i_{1}=k-1}^{n-1} \cdots \sum_{i_{k-1}=1}^{i_{k-2}-1}\left(\begin{array}{c}
n \\
i_{1}
\end{array}\right) \cdots\left(\begin{array}{c}
i_{k-2} \\
i_{k-1}
\end{array}\right)\left(\begin{array}{c}
i_{k-1} \\
0
\end{array}\right) \\
& \quad \times f\left(x_{1}^{n-i_{1}} \cdots x_{k-1}^{i_{k-2}-i_{k-1}} x_{k}^{i_{k-1}}\right) \\
& =\sum_{i_{1}=k-1}^{n-1} \cdots \sum_{i_{k-1}=1}^{i_{k-2}-1}\left(\begin{array}{c}
n \\
i_{1}
\end{array}\right) \cdots\left(\begin{array}{c}
i_{k-2} \\
i_{k-1}
\end{array}\right)\left(\begin{array}{c}
i_{k-1} \\
0
\end{array}\right) \\
& \quad \times f\left(x_{1}\right)^{n-i_{1}} \cdots f\left(x_{k-1}\right)^{i_{k-2}-i_{k-1}} f\left(x_{k}\right)^{i_{k-1}},
\end{aligned}
$$

for all $x_{1}, x_{2}, x_{3}, \ldots, x_{k} \in A$. Recall that the equality,

$$
\left(x_{k}+x_{k+1}\right)^{i_{k-1}}=\sum_{i_{k}=0}^{i_{k-1}}\left(\begin{array}{c}
i_{k-1} \\
i_{k}
\end{array}\right) x_{k}^{i_{k-1}-i_{k}} x_{k+1}^{i_{k}},
$$

holds for all $x_{k}, x_{k+1} \in A$. Replacing $x_{k}$ by $x_{k}+x_{k+1}$ in (3), we obtain

$$
\begin{aligned}
& \sum_{i_{1}=k-1}^{n-1} \cdots \sum_{i_{k-1}=1}^{i_{k-2}-1} \sum_{i_{k}=0}^{i_{k-1}}\left(\begin{array}{c}
n \\
i_{1}
\end{array}\right) \cdots\left(\begin{array}{c}
i_{k-2} \\
i_{k-1}
\end{array}\right)\left(\begin{array}{c}
i_{k-1} \\
i_{k}
\end{array}\right) \\
& \times f\left(x_{1}^{n-i_{1}} \cdots x_{k-1}^{i_{k-2}-i_{k-1}} x_{k}^{i_{k-1}-i_{k}} x_{k+1}^{i_{k}}\right) \\
& =\sum_{i_{1}=k-1}^{n-1} \sum_{i_{2}=k-2}^{i_{1}-1} \cdots \sum_{i_{k-1}=1}^{i_{k-2}-1}\left(\begin{array}{l}
n \\
i_{1}
\end{array}\right) \cdots\left(\begin{array}{l}
i_{k-2} \\
i_{k-1}
\end{array}\right) \\
& \times f\left(x_{1}^{n-i_{1}} \cdots x_{k-1}^{i_{k-2}-i_{k-1}}\right. \\
& \left.\times\left(x_{k}+x_{k+1}\right)^{i_{k-1}}\right) \\
& =\sum_{i_{1}=k-1 i_{2}=k-2}^{n-1} \sum_{i_{k-1}=1}^{i_{1}-1} \ldots \sum_{i_{k-2}-1}^{n}\left(\begin{array}{l}
n \\
i_{1}
\end{array}\right)\left(\begin{array}{l}
i_{1} \\
i_{2}
\end{array}\right) \cdots\left(\begin{array}{l}
i_{k-2} \\
i_{k-1}
\end{array}\right) \\
& \times f\left(x_{1}\right)^{n-i_{1}} \cdots f\left(x_{k}+x_{k+1}\right)^{i_{k-1}} \\
& =\sum_{i_{1}=k-1}^{n-1} \sum_{i_{2}=k-2}^{i_{1}-1} \cdots \sum_{i_{k-1}=1}^{i_{k-2}-1}\left(\begin{array}{l}
n \\
i_{1}
\end{array}\right)\left(\begin{array}{l}
i_{1} \\
i_{2}
\end{array}\right) \cdots\left(\begin{array}{l}
i_{k-2} \\
i_{k-1}
\end{array}\right) \\
& \times f\left(x_{1}\right)^{n-i_{1}} \cdots\left(f\left(x_{k}\right)+f\left(x_{k+1}\right)\right)^{i_{k-1}} \\
& =\sum_{i_{1}=k-1}^{n-1} \cdots \sum_{i_{k-1}=1}^{i_{k-2}-1} \sum_{i_{k}=0}^{i_{k-1}}\left(\begin{array}{l}
n \\
i_{1}
\end{array}\right) \cdots\left(\begin{array}{c}
i_{k-2} \\
i_{k-1}
\end{array}\right)\left(\begin{array}{c}
i_{k-1} \\
i_{k}
\end{array}\right) \\
& \times f\left(x_{1}\right)^{n-i_{1}} \cdots f\left(x_{k}\right)^{i_{k-1}-i_{k}} f\left(x_{k+1}\right)^{i_{k}},
\end{aligned}
$$

for all $x_{1}, x_{2}, x_{3}, \ldots, x_{k}, x_{k+1} \in A$. From (5), (6), and the above equality, we get the desired equality:

$$
\begin{aligned}
& \sum_{i_{1}=k}^{n-1} \sum_{i_{2}=k-1}^{i_{1}-1} \cdots \sum_{i_{k}=1}^{i_{k-1}-1}\left(\begin{array}{l}
n \\
i_{1}
\end{array}\right)\left(\begin{array}{c}
i_{1} \\
i_{2}
\end{array}\right) \cdots\left(\begin{array}{c}
i_{k-1} \\
i_{k}
\end{array}\right) \\
& \times f\left(x_{1}^{n-i_{1}} x_{2}^{i_{1}-i_{2}} \cdots x_{k+1}^{i_{k}}\right) \\
& =\sum_{i_{1}=k-1}^{n-1} \ldots \sum_{i_{k-1}=1}^{i_{k-2}-1} \sum_{i_{k}=0}^{i_{k-1}}\left(\begin{array}{c}
n \\
i_{1}
\end{array}\right) \cdots\left(\begin{array}{c}
i_{k-2} \\
i_{k-1}
\end{array}\right)\left(\begin{array}{c}
i_{k-1} \\
i_{k}
\end{array}\right) \\
& \times f\left(x_{1}^{n-i_{1}} \cdots x_{k-1}^{i_{k-2}-i_{k-1}}\right. \\
& \left.\times x_{k}^{i_{k-1}-i_{k}} x_{k+1}^{i_{k}}\right) \\
& -\sum_{i_{1}=k-1}^{n-1} \cdots \sum_{i_{k-1}=1}^{i_{k-2}-1}\left(\begin{array}{l}
n \\
i_{1}
\end{array}\right) \cdots\left(\begin{array}{l}
i_{k-2} \\
i_{k-1}
\end{array}\right)\left(\begin{array}{l}
i_{k-1} \\
i_{k-1}
\end{array}\right) \\
& \times f\left(x_{1}^{n-i_{1}} \cdots x_{k-1}^{i_{k-2}-i_{k-1}} x_{k+1}^{i_{k-1}}\right) \\
& -\sum_{i_{1}=k-1}^{n-1} \ldots \sum_{i_{k-1}=1}^{i_{k-2}-1}\left(\begin{array}{l}
n \\
i_{1}
\end{array}\right) \cdots\left(\begin{array}{l}
i_{k-2} \\
i_{k-1}
\end{array}\right)\left(\begin{array}{c}
i_{k-1} \\
0
\end{array}\right) \\
& \times f\left(x_{1}^{n-i_{1}} \cdots x_{k-1}^{i_{k-2}-i_{k-1}} x_{k}^{i_{k-1}}\right)
\end{aligned}
$$




$$
\begin{aligned}
& =\sum_{i_{1}=k-1}^{n-1} \cdots \sum_{i_{k-1}=1}^{i_{k-2}-1} \sum_{i_{k}=0}^{i_{k-1}}\left(\begin{array}{l}
n \\
i_{1}
\end{array}\right) \cdots\left(\begin{array}{c}
i_{k-2} \\
i_{k-1}
\end{array}\right)\left(\begin{array}{c}
i_{k-1} \\
i_{k}
\end{array}\right) \\
& \times f\left(x_{1}\right)^{n-i_{1}} \cdots f\left(x_{k}\right)^{i_{k-1}-i_{k}} \\
& \times f\left(x_{k+1}\right)^{i_{k}} \\
& -\sum_{i_{1}=k-1}^{n-1} \cdots \sum_{i_{k-1}=1}^{i_{k-2}-1}\left(\begin{array}{l}
n \\
i_{1}
\end{array}\right) \cdots\left(\begin{array}{l}
i_{k-2} \\
i_{k-1}
\end{array}\right)\left(\begin{array}{l}
i_{k-1} \\
i_{k-1}
\end{array}\right) \\
& \times f\left(x_{1}\right)^{n-i_{1}} \cdots f\left(x_{k-1}\right)^{i_{k-2}-i_{k-1}} \\
& \times f\left(x_{k+1}\right)^{i_{k-1}} \\
& -\sum_{i_{1}=k-1}^{n-1} \cdots \sum_{i_{k-1}=1}^{i_{k-2}-1}\left(\begin{array}{l}
n \\
i_{1}
\end{array}\right) \cdots\left(\begin{array}{l}
i_{k-2} \\
i_{k-1}
\end{array}\right)\left(\begin{array}{c}
i_{k-1} \\
0
\end{array}\right) \\
& \times f\left(x_{1}\right)^{n-i_{1}} \cdots f\left(x_{k-1}\right)^{i_{k-2}-i_{k-1}} \\
& \times f\left(x_{k}\right)^{i_{k-1}} \\
& =\sum_{i_{1}=k}^{n-1} \sum_{i_{2}=k-1}^{i_{1}-1} \cdots \sum_{i_{k}=1}^{i_{k-1}-1}\left(\begin{array}{l}
n \\
i_{1}
\end{array}\right)\left(\begin{array}{c}
i_{1} \\
i_{2}
\end{array}\right) \cdots\left(\begin{array}{c}
i_{k-1} \\
i_{k}
\end{array}\right) \\
& \times f\left(x_{1}\right)^{n-i_{1}} f\left(x_{2}\right)^{i_{1}-i_{2}} \cdots f\left(x_{k+1}\right)^{i_{k}},
\end{aligned}
$$

for all $x_{1}, x_{2}, x_{3}, \ldots, x_{k}, x_{k+1} \in A$.

The following theorem is the generalization of Theorem 1.

Theorem 4. Let $A, B$ be two commutative algebras, and let $f$ : $A \rightarrow B$ be an $n$-Jordan homomorphism. Then $f$ is an $n$-ring homomorphism.

Proof. Since $f$ is an $n$-Jordan homomorphism, together with the additivity of $f$, we get

$$
\begin{aligned}
\sum_{i=0}^{n}\left(\begin{array}{c}
n \\
i
\end{array}\right) f\left(x_{1}^{n-i} x_{2}^{i}\right) & =f\left(\left(x_{1}+x_{2}\right)^{n}\right)=f\left(x_{1}+x_{2}\right)^{n} \\
& =\left(f\left(x_{1}\right)+f\left(x_{2}\right)\right)^{n} \\
& =\sum_{i=0}^{n}\left(\begin{array}{c}
n \\
i
\end{array}\right) f\left(x_{1}\right)^{n-i} f\left(x_{2}\right)^{i}
\end{aligned}
$$

for all $x_{1}, x_{2} \in A$. It is clear that $f\left(x_{1}^{n}\right)=f\left(x_{1}\right)^{n}$ and $f\left(x_{2}^{n}\right)=$ $f\left(x_{2}\right)^{n}$, so we obtain

$$
\sum_{i=1}^{n-1}\left(\begin{array}{c}
n \\
i
\end{array}\right) f\left(x_{1}^{n-i} x_{2}^{i}\right)=\sum_{i=1}^{n-1}\left(\begin{array}{c}
n \\
i
\end{array}\right) f\left(x_{1}\right)^{n-i} f\left(x_{2}\right)^{i}
$$

for all $x_{1}, x_{2} \in A$. If $n=2$, then by (11) we have $f\left(x_{1} x_{2}\right)=$ $f\left(x_{1}\right) f\left(x_{2}\right)$. Now let $n>2$. Together with Lemma 3 and (11), we can say that the equality (4) holds for $k=n-1$; that is,

$$
\begin{aligned}
& \sum_{i_{1}=n-1}^{n-1} \sum_{i_{2}=n-2}^{i_{1}-1} \ldots \sum_{i_{n-1}=1}^{i_{n-2}-1}\left(\begin{array}{l}
n \\
i_{1}
\end{array}\right)\left(\begin{array}{l}
i_{1} \\
i_{2}
\end{array}\right) \cdots\left(\begin{array}{l}
i_{n-2} \\
i_{n-1}
\end{array}\right) \\
& \times f\left(x_{1}^{n-i_{1}} x_{2}^{i_{1}-i_{2}} x_{3}^{i_{2}-i_{3}} \cdots x_{n}^{i_{n-1}}\right) \\
&=\sum_{i_{1}=n-1}^{n-1} \sum_{i_{2}=n-2}^{i_{1}-1} \cdots \sum_{i_{n-1}=1}^{i_{n-2}-1}\left(\begin{array}{l}
n \\
i_{1}
\end{array}\right)\left(\begin{array}{l}
i_{1} \\
i_{2}
\end{array}\right) \cdots\left(\begin{array}{l}
i_{n-2} \\
i_{n-1}
\end{array}\right) \\
& \times f\left(x_{1}\right)^{n-i_{1}} f\left(x_{2}\right)^{i_{1}-i_{2}} \cdots f\left(x_{n}\right)^{i_{n-1}},
\end{aligned}
$$

holds for all $x_{1}, x_{2}, x_{3}, \ldots, x_{n} \in A$. Notice that

$$
n-1>i_{1}>i_{2}>\cdots>i_{n-2}>i_{n-1} \geq 1
$$

implies $i_{1}=n-1, i_{2}=n-2, \ldots, i_{n-2}=2, i_{n-1}=1$ and so

$$
n-i_{1}, i_{1}-i_{2}, \ldots, i_{n-1}-i_{n-2}, i_{n-1}=1 \text {. }
$$

Therefore we get the desired equality:

$$
f\left(x_{1} x_{2} x_{3} \cdots x_{n}\right)=f\left(x_{1}\right) f\left(x_{2}\right) f\left(x_{3}\right) \cdots f\left(x_{n}\right)
$$

for all $x_{1}, x_{2}, x_{3}, \ldots, x_{n} \in A$.

\section{Generalization of Theorem 2}

We need the following lemmas to prove the generalization of Theorem 2.

Lemma 5 (see [9, Corollaries 2.5 and 3.5]). Let $V$ be a normed space, and let $W$ be a Banach space. Assume that $f, g, h: V \rightarrow$ $W$ are mappings such that

$$
\|f(x+y)-g(x)-h(y)\| \leq \varepsilon\left(\|x\|^{p}+\|y\|^{p}\right),
$$

for all $x, y \in V \backslash\{0\}$, where $p \neq 1$ and $\varepsilon \geq 0$. Then there exists a unique additive mapping $T: V \rightarrow W$ such that

$$
\|f(x)-T(x)-f(0)\| \leq \frac{\left|4\left(3+3^{p}\right)\right| \varepsilon}{\left|2^{p}\left(3-3^{p}\right)\right|}\|x\|^{p},
$$

for all $x \in V \backslash\{0\}$. In particular, $T$ is given by

$$
T(x)=\lim _{m \rightarrow \infty} \frac{f\left(3^{s m} x\right)-f(0)}{3^{s m}},
$$

for all $x \in V \backslash\{0\}$, where $s:=-\operatorname{sgn}(p-1)$.

Lemma 6. Let $V, W, f, g, h, \varepsilon$ be as in Lemma 5. If $p<0$ and $f(0)=0$, then $f$ is an additive mapping. 
Proof. Let $T: V \rightarrow W$ be the additive mapping satisfying (17). Then we have

$$
\begin{aligned}
\|2 f(x)-2 T(x)\| \leq & \|f(2(n+1) x)-T(2(n+1) x)\| \\
& +\|f(-2 n x)-T(-2 n x)\| \\
& +\|f(x)-g((n+1) x)-h(-n x)\| \\
& +\|f(x)-g(-n x)-h((n+1) x)\| \\
& +\| f(2(n+1) x) \\
& +\|f(-2 n x)-g(-n x)-h(-n x)\| \\
\leq & \left(\frac{4\left(3+3^{p}\right)}{2^{p}\left(3-3^{p}\right)}+4\right)(n+1)^{p} \varepsilon\|x\|^{p} \\
& +\left(\frac{4\left(3+3^{p}\right)}{2^{p}\left(3-3^{p}\right)}+4\right) n^{p} \varepsilon\|x\|^{p},
\end{aligned}
$$

for all $x \in V \backslash\{0\}$ and $n \in \mathbb{N}$. Taking the limit as $n \rightarrow \infty$, we get $f(x)=T(x)$ as desired.

The following result has already been proved in [7] (see also [8]). We show that it can also be derived from Lemma 6.

Lemma 7. Let $V, W, \varepsilon$ be as in Lemma 5 and $p<0$. If $f: V \rightarrow$ $W$ is a mapping such that

$$
\begin{array}{r}
\|f(x+y)-f(x)-f(y)\| \leq \varepsilon\left(\|x\|^{p}+\|y\|^{p}\right) \\
\forall x, y \in V \backslash\{0\},
\end{array}
$$

then $f$ is an additive mapping.

Proof. By Lemma 5, we can take an additive mapping $T$ : $V \rightarrow W$ satisfying (17). Observe that

$$
\begin{aligned}
\|f(0)\| \leq & \|f(n x)-T(n x)-f(0)\| \\
& +\|f(-n x)-T(-n x)-f(0)\| \\
& +\|f(0)-f(n x)-f(-n x)\| \\
\leq & \left(\frac{8\left(3+3^{p}\right)}{2^{p}\left(3-3^{p}\right)}+2\right) n^{p} \varepsilon\|x\|^{p},
\end{aligned}
$$

for all $x \in V \backslash\{0\}$ and for all $n \in \mathbb{N}$. Taking the limit as $n \rightarrow$ $\infty$, we get $f(0)=0$. By Lemma $6, f$ is an additive mapping.

Now we can prove the following theorem which is the generalization of Theorem 2 .

Theorem 8. Let $A$ be a commutative normed algebra and $B$ a commutative Banach algebra. Assume that $f, g, h: A \rightarrow B$ satisfy (16) and

$$
\left\|f\left(x^{n}\right)-f(x)^{n}\right\| \leq \delta\|x\|^{n q},
$$

for all $x \in A \backslash\{0\}$, where $\delta \geq 0$ and $(p-1)(q-1)>0$. If $f(0)=0$, then there exists a unique n-ring homomorphism $T: A \rightarrow B$ satisfying (17).

Proof. By Lemma 5, there exists a unique additive mapping $T$ satisfying (17). By Theorem 4 , it suffices to show that $T\left(x^{n}\right)=$ $T(x)^{n}$. Put $s:=-\operatorname{sgn}(q-1)$. From the equality below (17) in Lemma 5, we have

$$
T(x)=\lim _{m \rightarrow \infty} \frac{f\left(3^{s m} x\right)}{3^{s m}},
$$

for all $x \in A \backslash\{0\}$. It follows from (22) that

$$
\begin{aligned}
\left\|T\left(x^{n}\right)-T(x)^{n}\right\| & =\lim _{m \rightarrow \infty} \frac{1}{3^{s m n}}\left\{\left\|f\left(\left(3^{s m} x\right)^{n}\right)-\left(f\left(3^{s m} x\right)\right)^{n}\right\|\right\} \\
& \leq \lim _{m \rightarrow \infty} \frac{\delta}{3^{s m n}}\left\|3^{s m} x\right\|^{n q} \\
& =\lim _{m \rightarrow \infty}\left(3^{s m n(q-1)}\right) \delta\|x\|^{n q}=0,
\end{aligned}
$$

for all $x \in A \backslash\{0\}$. Hence $T$ is an $n$-Jordan homomorphism. By Theorem 4, $T$ is an $n$-ring homomorphism.

The following two corollaries give results on the hyperstability of $n$-ring homomorphisms between Banach algebras.

Corollary 9. Let $A, B, q, \delta, f, g, h$ be as in Theorem 8 . If $f(0)=0$ and $p<0$, then $f$ is an n-ring homomorphism.

Proof. Let $T$ be the unique $n$-ring homomorphism satisfying (17) in Theorem 8. By Lemma 6, $f$ is the unique additive mapping satisfying (17). So $f$ is the unique $n$-ring homomorphism.

Corollary 10. Let $A, B, p, q$ be as in Corollary 9. Assume that $f: A \rightarrow B$ satisfies the system of functional inequalities (20) and (22) for all $x \in A \backslash\{0\}$. Then $f$ is ann-ring homomorphism.

Proof. The proof is analogous as for Corollary 9, with Lemma 6 replaced by Lemma 7.

\section{Acknowledgments}

The author thanks the reviewers and the editor for their very helpful comments that improve the paper. This research was supported by Basic Science Research Program through the National Research Foundation of Korea (NRF) funded by the Ministry of Education, Science and Technology (no. 2012R1A1A4A01002971).

\section{References}

[1] M. E. Gordji, T. Karimi, and S. K. Gharetapeh, "Approximately $n$-Jordan Homomorphisms on Banach algebras," Journal of Inequalities and Applications, vol. 2009, Article ID 870843, 8 pages, 2009.

[2] S. M. Ulam, Problems in Modern Mathematics, chapter 6, John Wiley \& Sons, New York, NY, USA, 1964. 
[3] D. G. Bourgin, "Approximately isometric and multiplicative transformations on continuous function rings," Duke Mathematical Journal, vol. 16, pp. 385-397, 1949.

[4] R. Badora, "On approximate derivations," Mathematical Inequalities \& Applications, vol. 9, no. 1, pp. 167-173, 2006.

[5] N. Brillouët-Belluot, J. Brzdęk, and K. Ciepliński, "On some recent developments in Ulam's type stability," Abstract and Applied Analysis, vol. 2012, Article ID 716936, 41 pages, 2012.

[6] G. Maksa and Z. Páles, "Hyperstability of a class of linear functional equations," Acta Mathematica, vol. 17, no. 2, pp. 107$112,2001$.

[7] J. Brzdek, "Hyperstability of the Cauchy equation on restricted domains," Acta Mathematica Hungarica, 2013.

[8] M. Piszczek, "Remark on hyperstability of the general linear equation," Aequationes Mathematicae, 2013.

[9] Y.-H. Lee and K.-W. Jun, "A generalization of the HyersUlam-Rassias stability of the Pexider equation," Journal of Mathematical Analysis and Applications, vol. 246, no. 2, pp. 627$638,2000$. 


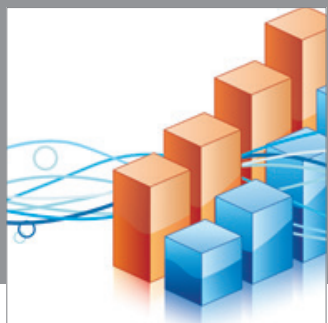

Advances in

Operations Research

mansans

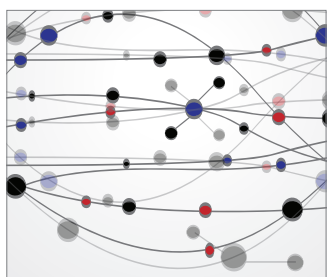

The Scientific World Journal
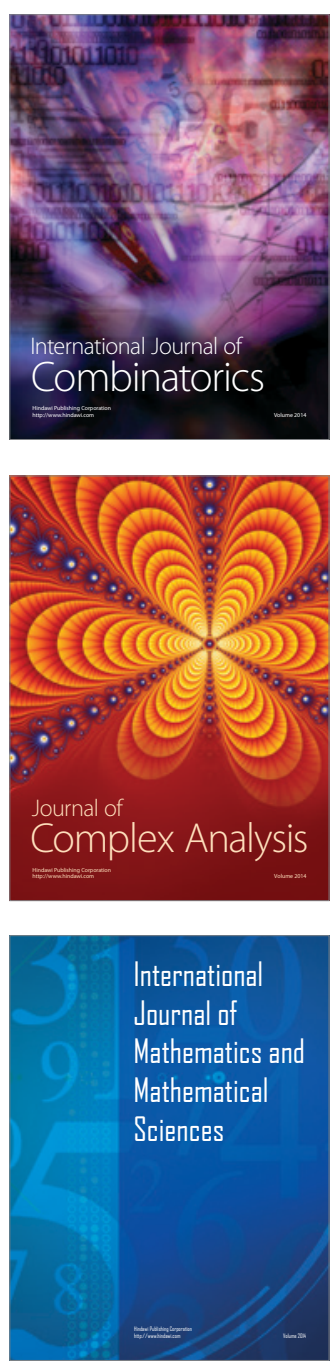
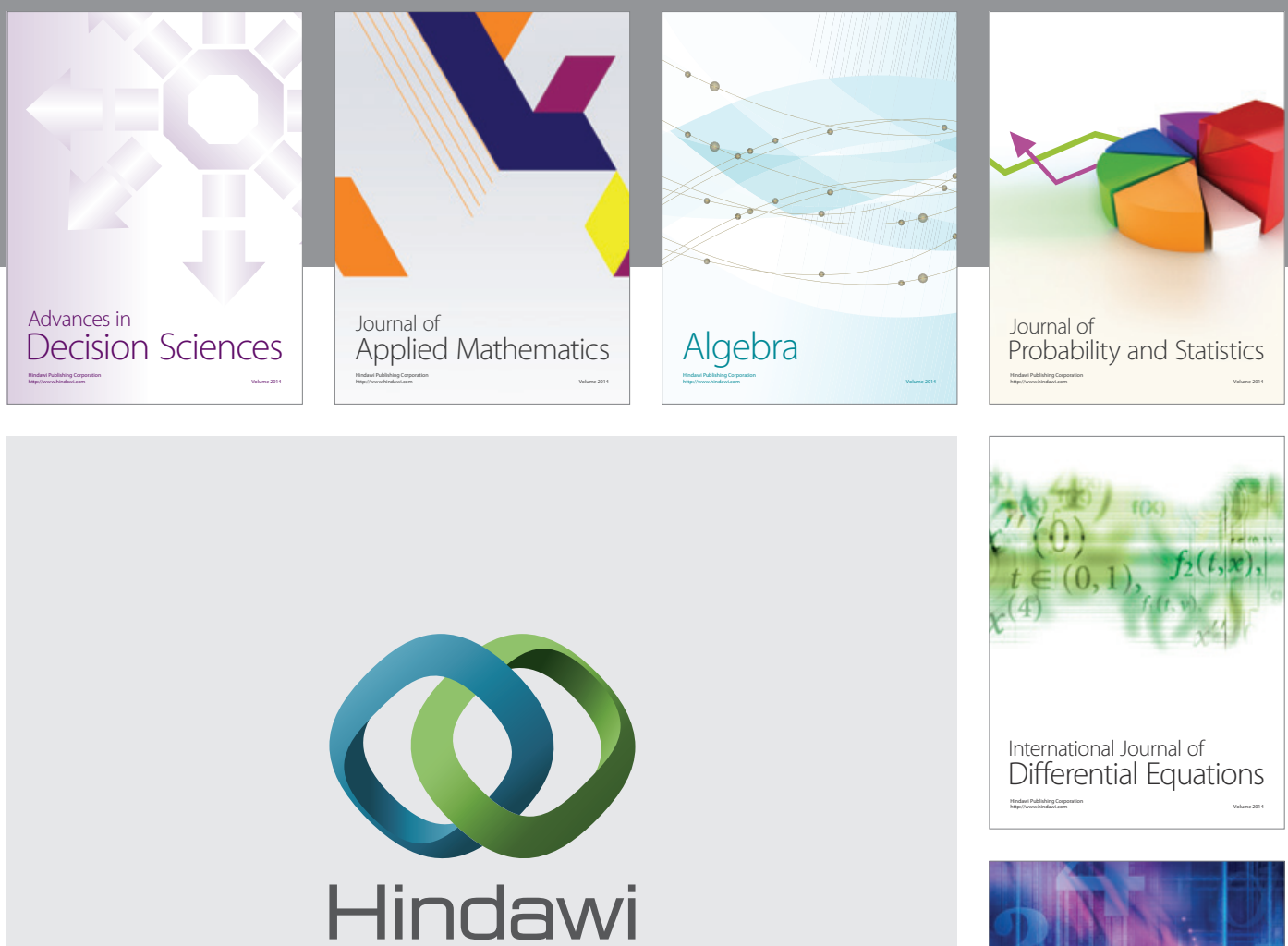

Submit your manuscripts at http://www.hindawi.com
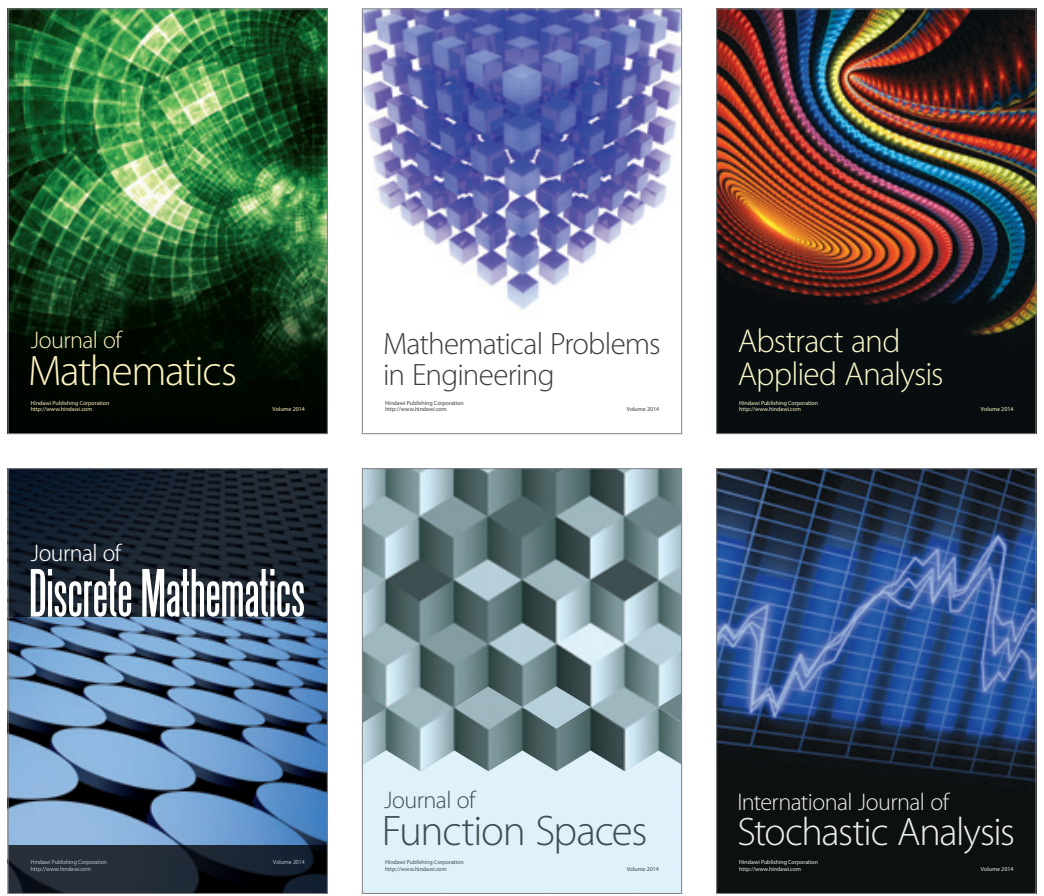

Journal of

Function Spaces

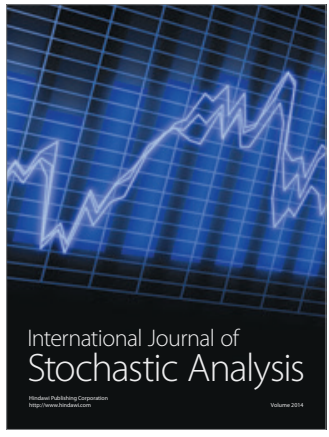

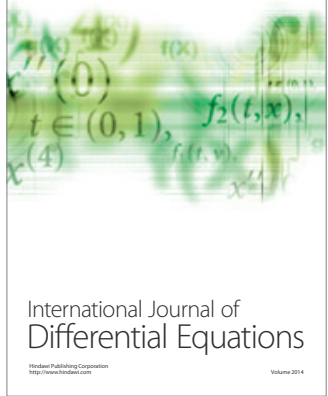
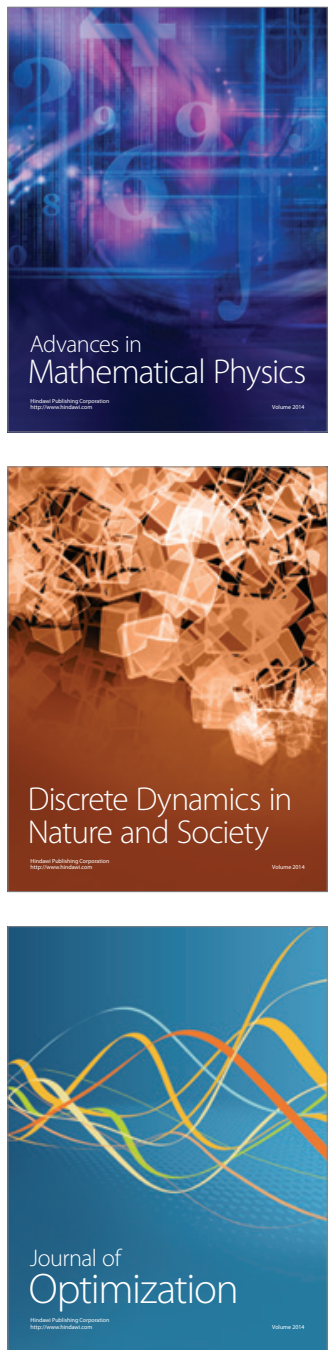